\begin{tabular}{|c|c|}
\hline Title & The association between colors and emotions for emotional words and facial expressions \\
\hline Author(s) & Takahashi, Fumiyo; Kawabata, Y asuhiro \\
\hline Citation & $\begin{array}{l}\text { Color research and application, 43(2), 247-257 } \\
\text { https://doi.org/10.1002/col.22186 }\end{array}$ \\
\hline Issue Date & $2018-04$ \\
\hline Doc URL & http://hdl.handle.net/2115/71652 \\
\hline Rights & $\begin{array}{l}\text { This is the peer reviewed version of the following article: [The association between colors and emotions for emotional } \\
\text { words and facial expressions], which has been published in final form at [https://doi.org/10.1002/col.22186]. This } \\
\text { article may be used for non-commercial purposes in accordance with Wiley Terms and Conditions for Self-A rchiving. }\end{array}$ \\
\hline Type & article (author version) \\
\hline File Information & revision170814latest_r180327_text-legend-replace.pdf \\
\hline
\end{tabular}

Instructions for use 


\title{
The Association between Colors and Emotions for Emotional Words and Facial Expressions.
}

\author{
Fumiyo Takahashi $^{1}$, Yasuhiro Kawabata ${ }^{1}$ \\ ${ }^{1}$ Department of Psychology, Hokkaido University, Sapporo, Japan
}

\begin{abstract}
Color sensations are tied not only to other sensations but also emotions. There have been many studies on this. One study regarding architectural color showed that colors were associated with mental status; for example, red relates to arousal, excitation, and stimulus. ${ }^{1}$ The purpose of the present study is to investigate how colors are evoked by emotions. The emotions were described both by emotional words and by schematic faces. Since facial expressions are accompanied by facial color, facial expressions should relate more closely to facial color than emotional words. Therefore, we used numerous color samples for our experiments to show discrimination sensitivity to stimuli in subtle differences of color. Some associations between colors and emotions were found, and the tendencies of associations were different among emotions. Anger, joy, surprise, sadness, and no emotion were connected to particular colors. The distribution of color responses in sadness was spread among bluish colors. The emotional tendencies, among anger, joy, surprise, and sadness, were similar in the two conditions of our experiment. However, in the schematic face condition, the color responses for all emotions were increased in the skin-colored samples. Thus, the context of the face elicited the color responses.
\end{abstract}

Keywords: color emotion, emotional word, facial expression, facial color, psychological evaluation

\section{Introduction}

Colors tend to be related to other sensations and emotions. Previous research on the association between colors and emotions used pairs of terms to describe sensations, such as warm-cool, hard-soft, and heavy-light. ${ }^{1,2,3,4}$ The "hue-heat" hypothesis, which describes the connection between colors and sensations of temperature, is that an environment with dominant red frequencies feels warm and one with dominant blue frequencies feels cool ${ }^{5}$. Color sensations may connect not only to other sensations but also emotions. Past research on architectural color showed that colors are associated with mental status, such as red, relating to arousal, excitation, and stimulus; orange, being lively, energetic, and extroverted; yellow, representing hope, wisdom, and expansive; green, being refreshing, quiet, and natural; blue, evoking passivity, cleanliness, quietness, etc. ${ }^{1,6}$ The purpose of this study is to determine how colors are evoked by emotions. The emotions were described both by schematic faces and by emotional words. Since facial expressions are accompanied by facial color, facial expressions will more closely relate to facial color than emotional words. Therefore, we used numerous color samples for our experiments to show discrimination sensitivity in subtle difference of color to stimuli. However, cognitive processes for words are different from those for color. Hence, the present study has two aims.

First, we investigate the association of facial expressions with colors by using schematic faces. This is based on Mollon's hypothesis. Mollon (1989) ${ }^{7}$ hypothesized that trichromatic vision in primates including human beings, was selected for to discriminate the spectral modulations on the skin of conspecifics. Primates signal sexual readiness through changing colors on their faces or genitals, and trichromatic vision could be generated to 
distinguish changes in skin colors. ${ }^{7}$ Changizi et al. (2006) ${ }^{8}$ supported this hypothesis by computer modeling, noting that trichromatic primates tend to be bare faced. Facial expressions with skin colors are important as social signals. Many studies of the recognition of facial expressions also support this hypothesis. Suzuki and Koyatsu (1996) ${ }^{9}$ showed that changing facial colors influenced on the judgment of facial expressions: facial expressions of happiness were evaluated as being happier with the increasing redness of the skin color; as for sadness, it was evaluated as sadder with increasing blueness. Based on these studies, we considered that people will discriminate subtle differences of color for each emotional facial expression. Thus, we considered that colors associated with emotional complexion are more direct.

Second, we investigated the association between emotional words and colors. There are many phrases using colors for expressing emotions in Japanese language, such as, to get angry with a red face (to see red), to become white in one's head (one's head goes blank), one's face turns bluish white (to turn pale, white as a sheet), to be red with pleasure (to flush with pleasure, to be tickled pink), to yell in a yellow voice (to yell in a high-pitched voice), and to feel blue. We assume that certain emotional words are associated with color. Since emotional words are more abstract than schematic faces, more targets that are associated with something besides facial expressions, could be evoked in order to image colors. We showed participants emotional words corresponding to emotional facial expressions. Participants selected imaged colors, that fit an emotional word from a color palette. Many previous studies on color emotion have used emotional words. ${ }^{10-15}$ Byrnes $(1983)^{10}$ showed a predominant color association among children in the cases of love to red, death to black, and anger to red, closely followed by black. Various colors were chosen for happiness. Overall, it was found that these color choices were similar to those reported in adults. Another study in children used drawings and showed that they have distinct emotional associations with color. Brighter colors such as orange, yellow, green, and blue, are given as responses for association with positive emotion. On the other hand, brown, black, and red are given as responses for dramatic effects. ${ }^{16}$ Studies on adults demonstrated similar results for brightness. Relatively brighter colors, such as white, pink, red, yellow, blue, purple, and green, evoked positive responses, while darker ones, such as black and gray, evoked negative responses. Positive responses were related to happiness, excitement, relaxation, and being positive. Negative responses were related to anxiety, boredom, sadness, and being negative. ${ }^{17,18}$ A study in college students showed that positive emotional responses were the highest in five principal colors (red, yellow, green, blue, purple), followed by intermediate colors (yellow-red, green-yellow, blue-green, purple-blue, red-purple) and achromatic colors (white, gray, black) ${ }^{19}$. Green evoked mainly positive emotions such as relaxation and comfort, and green-yellow had the lowest number of positive responses. Among the achromatic colors, white had the largest number of positive responses followed by black and gray. ${ }^{1,19}$ Thus, the association between color and emotion is shown from the previous studies.

From these facts, we presume that the results will differ depending on the difference in abstraction level of stimuli. In other words, the color association in the face condition will be rather limited to facial colors, and the association in the word condition will be more various colors. Accordingly, we assume that there is a contextual effect of face in the face condition as the participants' response will increase in skin colors or similar colors. Furthermore, the originality of our research is to use a color palette of 130 color samples with various hues, saturations, and brightness. Although only a few color samples have been used in past studies on facial expressions, ${ }^{9,20}$ we considered that using more color samples could reduce the gap between an actual continuous color space and experimental stimuli. We conducted our experiments using color naming in an analogous way, though many previous studies used a semantic differential method. ${ }^{1,3,4}$ Using our color palette, which has subtle gradations of colors, enabled further analysis of the distribution of color responses in these conditions, and then the association between color and stimuli was analyzed. Thus, we investigated the association between colors and emotions in our two conditions of emotional words and schematic faces. We used basic emotions ${ }^{21}$ as stimuli in both conditions. We named 
them respectively the word condition and face condition. Thus, we compared the responses between the conditions.

\section{Method}

\section{Apparatus and stimuli}

The color palette used in our experiment was presented on a 19-inch LCD display (Sony, SDM-S93/HK) with $60-\mathrm{Hz}$ refresh rate and 1,280 × 1,024pixel spatial resolution. The top, back, left and right sides of the display were covered with black paper and the room lights were adjusted so that the effect of the light on the display was below threshold. The samples in the color palette were calibrated by chromatic photometers (Minolta, CS-100; Minolta, CA-100).

The color palette was designed based on the Practical Color Co-ordinates System (PCCS). PCCS, Japanese color appearance system, is composed of 24 hues and 12 tones. It was developed as an educational color atlas to show relations between colors in terms of color harmony. The PCCS's tone index consists of lightness (we describe brightness because a monitor a luminous source was used in our experiments) and saturation. It defines color variations by hue and tone such as pale, light, light grayish, soft, bright, strong, vivid, dull, grayish, deep, dark and dark grayish. When we perceive a color, we are not conscious of three attributes: hue, brightness and saturation, instead, we recognize color based on tone as we describe "dark green", "bright red”, etc. Therefore, color tone could be useful to discriminate a color easily. Thus, PCCS may enable participants to find colors efficiently from the palette.

The 130 samples include six focal colors (red, orange, yellow, green, blue and purple), and they were adjusted perceptually equal space on the basis of hue, brightness and saturation. The 130 samples were set in matrix state with 13 columns (1-13) $\times 10$ lines (A-J) so that participants could see all samples on the display at once. Each sample was placed with enough distances to the other samples not to cause the Herman Grid Illusion. Some similar hues were integrated and tuned adequately, and tones with brightness and saturation were tuned to ten stages in perceptually equal space on each hue. As a result, all lines were not totally congruent with the tones of PCCS. The columns showed hue: purple $(P)$, purplish blue $(p B)$, blue $(B)$, blue green $(B G)$, green $(G)$, yellowish green $(y G)$, greenish yellow $(g Y)$, yellow $(Y)$, orange $(O)$, yellowish red $(y R)$, red $(R)$, reddish purple $(r P)$, and grey scale $(B / W)$. The lines showed tones such as the brightest samples with low saturation at the top, the most saturated ones in the middle, and the darkest ones with low saturation at the bottom (Fig. 1). The color palette was presented on an LCD display. The size of each sample on the palette was visual angle of $1.2^{\circ}-1.5^{\circ}$. The display was viewed in a dimmed booth at a viewing distance of approximately 55 to $70 \mathrm{~cm}$. The background of samples was set in grey and its average of CIE points at $\mathrm{x}=.329, \mathrm{y}=.326, \mathrm{Y}=72.0$. Each six focal colors' PCCS value (hue-brightness-saturation) and location on the palette (line-column) was as follows, red: R-4.5-9s, 5-R; orange: O-6.5-9s, 5-O; yellow: Y-8.0-9s, 4-Y; green: G-5.5-9s, 6-BG; blue: B-3.5-9s, 7-B; purple: P-3.5-9s, 7-P. They were set on the palette. Each focal color was adjusted as closely as possible to the CIE values of PCCS's color paper sample which was measured with a photometer. CS- $100^{* 1}$. They were distributed overall with adequate distance in CIE color space (Fig. 2).

We designed the set of schematic faces to represent seven emotional facial expressions for schematic face condition. They were six basic emotions based on Ekman's face expressions (1969) ${ }^{22}$ and neutral status (Fig. 3). There are three reasons why we designed original schematic faces. First, prior researches showed almost the same emotional effect by schematic faces ${ }^{23-29}$ as by real faces. ${ }^{30}$ Second, we do not need to consider the gender

\footnotetext{
*1 Table 1 in the Appendix shows the CIE values of the samples on the display, as measured by a photometer, CA-100.
} 
in stimuli. Third, schematic faces used in previous researches were not familiar with Japanese people. Our pilot experiment showed that our stimuli were identified better than prior research's stimuli. ${ }^{31}$ The seven emotional words corresponding to the basic emotions were presented in Japanese words as follows, "ikari" as anger; "kanashimi” as sadness; "yorokobi” as joy; "ken-o" as disgust; "odoroki” as surprise; "Osore” as fear; "Mu-kanjo" as no emotion. All schematic faces used in face condition, were drawn with black line on white background with the same diameters of face outlines of $7.7 \mathrm{~cm}$. The experiment consisted of two blocks, emotional words’ block and emotional schematic faces’ block.

\section{Participants}

Forty Japanese students and staffs in Hokkaido University participated in the experiment. All had normal or corrected-to-normal visual acuity and normal color vision. They were divided into two groups, twenty for each group, to counterbalance the order of blocks. In first group, there were 10 males (21-28 years, $M=24.2, S D=$ 1.8 ) and 10 females (20-29 years, $M=23.5, S D=3.0$ ). In second group, there were 10 males (23-36 years, $M=$ 26.8, $S D=4.8$ ) and 10 females (21-34years, $M=26.1, S D=4.5)$. The word condition was conducted first and followed by the face condition for the first group, and vice versa for the second group. They were given their informed consent basis at the beginning of the experiments.

\section{Procedure}

In both word and face conditions, participants appraised color association for seven emotional stimuli respectively. The procedures were as follows. Experimenter first presented a summary of the experiment to participant and handed him/her answer sheets for word condition. Each emotional word was shown on the answer sheets. Experimenter then asked participant to imagine colors associated with the word. After participant confirmed that he/she already imagined colors, experimenter presented the palette on the display. Participant told experimenter what color he/she imagined, and pointed at the appropriate samples of the palette. The number of samples to choose were not limited. Experimenter marked the chosen samples on the display by Photoshop, and participant wrote down the marked colors on the answer sheet. Participants marked colors so as to derive $100 \%$ in total, according to the intensity of the image. For example, if participant selected four samples (The location of samples on the palette is indicated in line-column), E-2, E-3, E-8 and F-8 for the word disgust, participant might weigh the association as follows; $E-2(70 \%)+E-3(20 \%)+E-8(5 \%)+F-8(5 \%)=(100 \%)$. Only in word condition, if participants thought of some objects to imagine colors, they were asked to write down the items on the answer sheets (cf. Table3 in the Appendix). The order of presenting seven emotional words was randomized among participants. After participants had completed the initial condition, they took a rest between conditions if they needed. In both conditions, experimenter cleared marks on the palette by trials. In the face condition, a schematic face was presented on a board, and a participant was asked to imagine the facial color of the schematic face. The same procedures as the word condition were applied to a set of faces. The order of presenting the seven faces was randomized among participants. 


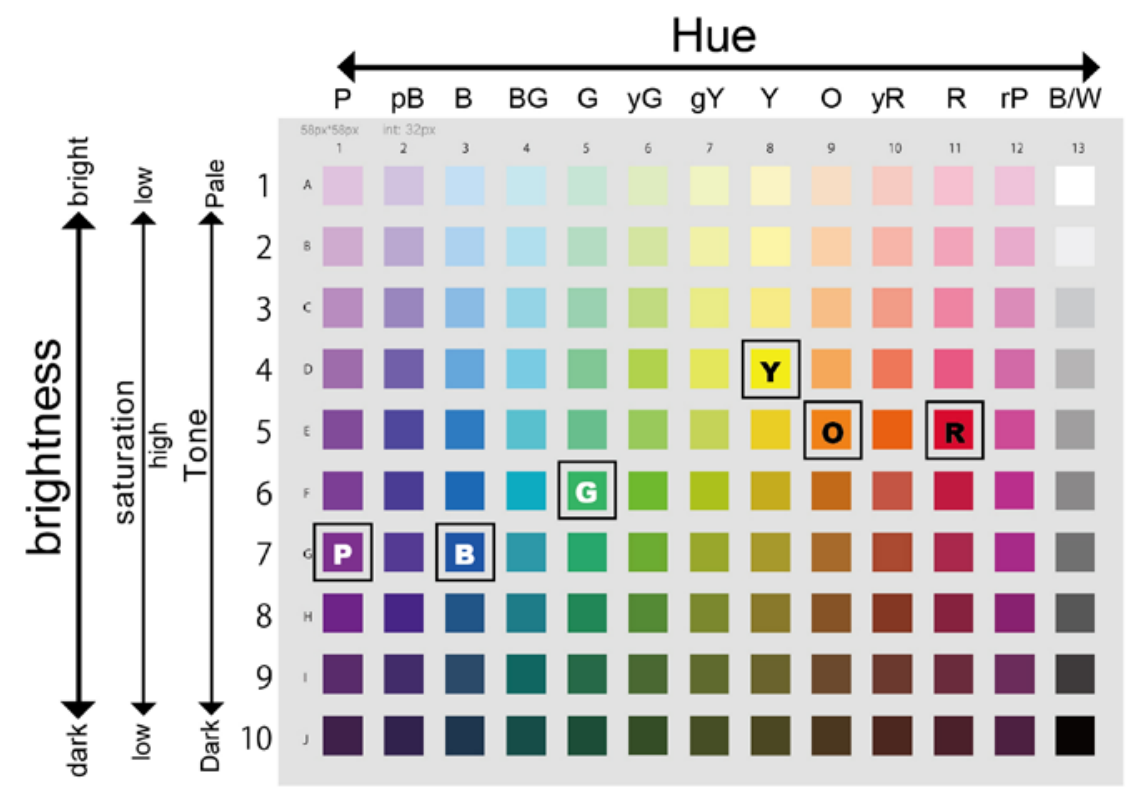

Fig. 1. The color palette used in the experiments. (left)

The color palette includes 130 color samples. Six of them, which are surrounded by squares and are in upper case, indicate focal colors. These uppercase letters stand for color names; $P$, purple; $B$, blue; $G$, green; $Y$, yellow; $O$, orange; and $R$, red.

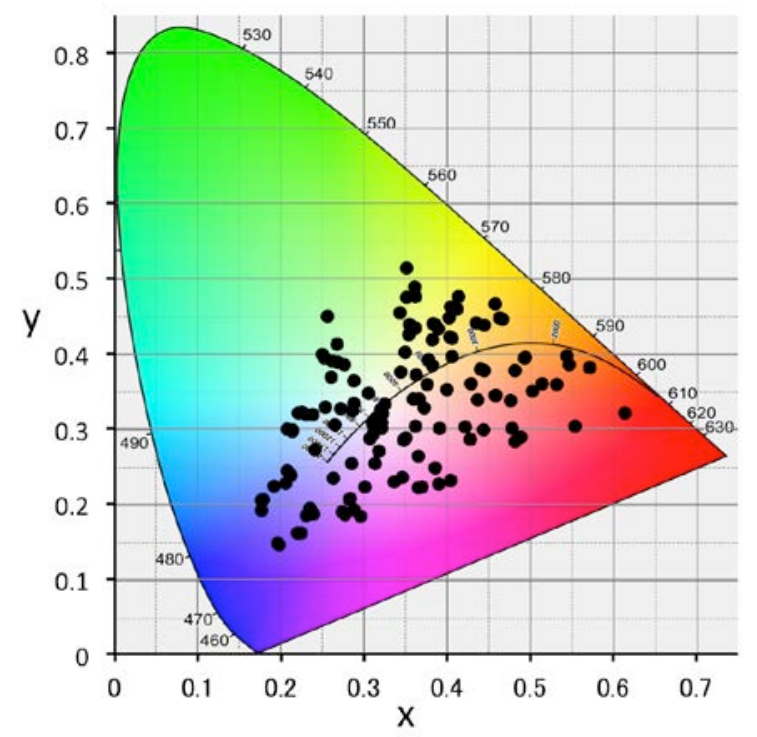

Fig. 2. The color samples in the CIE (x, y) color space.

The black spots show the measured CIE value (x, y) of the color samples on the palette. This shows that the color samples are appropriately adjusted to be dispersed in a balanced manner in the CIE color space.

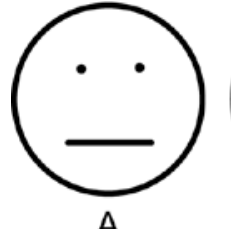

no emotion

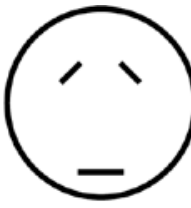

B

sadness

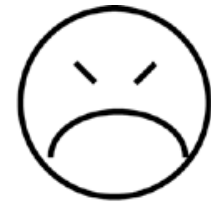

C

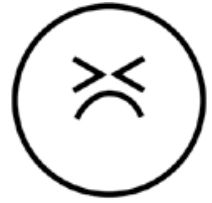

D

disgust

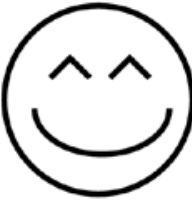

E

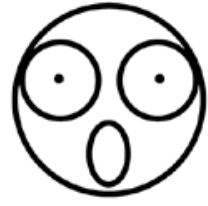

$\mathrm{F}$ surprise

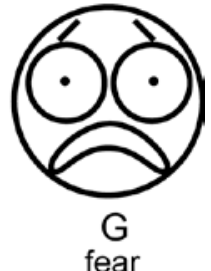

fear

Fig. 3. The schematic faces with emotional expressions for the stimuli of the face condition. 


\section{Results}

The experimental data were given in percentage. These were averages of weighted frequency that participants chose the color samples for each emotion of words and of schematic faces. We named these data "color response". We analyzed the first block's data of each stimulus set to compare the difference of color response between stimulus sets. The reason why we analyzed first block is the second block could have been biased by the first block. However, we used the first and second blocks' data on 40 participants for two-way repeated-measures ANOVA. An alpha level of .05 was used for all statistical tests.

\section{The association between color and emotion}

Fig. 4 shows the results of color response for each emotion of word conditions and of face conditions. In these figures, the vertical axis (z-axis) indicates the average of frequency on color response, and two horizontal axes (the x-axis: left to right; the y-axis: front to back) indicate the array of color samples on the palette which is shown in Fig. 1. Accordingly, the x-axis indicates hues, and the y-axis indicates brightness. The left side of the figures show the responses for word conditions, and the right side ones show the responses for face conditions. Each line gives the results of emotion. From the top, (a) anger, (b) Joy, (c) surprise and (d) no emotion, (e) sadness, (f) disgust and (g) fear. The parentheses show the results of Pearson's Correlation Analysis between conditions for each emotion. They were calculated by R language version 2.10.0. We consider that a high correlation coefficient means a high similarity between the conditions.

Based on the results, in anger, joy and surprise, the word conditions are strongly related to the face conditions, anger: $r(128)=.966, p<.001$; joy: $r(128)=.882, p<.001$; surprise: $r(128)=.695, p<.001$. In no emotion, the word conditions are moderately related to the face condition, $r(128)=.450, p<.001$. In these emotion, each graph has a clear peak in both conditions. We depict the detail of results for each emotion as below. Anger: modes of both conditions (word: $49.30 \%$; face: $47.33 \%$ ) were at the same color sample, red of focal color (5- $R$ on the palette). Repeated-measures ANOVA revealed main effects of brightness (word condition: $F$ [2.84, $110.71]=21.76, p<.001$; face condition: $F[3.12,121.55]=34.66, p<.001$ ) and of hue in both conditions (word: $F[2.56,99.83]=69.05, p<.001$; face: $F[2.99,116.63]=50.33, p<.001)$. Interaction brightness $\times$ hue was also significant in both conditions (word: $F[3.51,136.94]=22.08, p<.001$; face: $F[3.98,155.17]=27.11, p<.001$ ). In pairwise comparisons of both conditions for brightness, commonly brightness 5 was significantly higher than the remaining brightness degrees. Post hoc analyses were performed on brightness 5 by one-way repeated-measures ANOVA. As a result, 5- $R$ was significantly higher than all the rest (the main effect of hue in word condition, $F$ [1.18. 45.93] = 47.98, $p<.001$; in face condition: $F$ [1.39. 54.00] =52.42, $p$ <.001). Joy: modes of both conditions (word: $29.38 \%$; face: $27.18 \%$ ) were at the same color sample, yellow of focal color (4- $Y$ on the palette). Repeated-measures ANOVA revealed main effects of brightness (word: $F[3.19,124.48]=22.45, p$ $<.001$; face: $F[2.77,107.89]=18.91, p<.001$ ) and of hue in both conditions (word condition: $F[4.30,167.69]$ $=14.10, p<.001$; face condition: $F[3.14,122.27]=18.67, p<.001)$. Interaction brightness $\times$ hue was also significant in both conditions (word: $F[5.38,209.69]=10.26, p<.001$; face: $F[5.66,220.79]=10.59, p<.001$ ). In pairwise comparisons of both conditions for brightness, commonly brightnesses 4 was significantly higher than most of brightness degree. Post hoc analyses were performed on brightness 4 by one-way repeated-measures ANOVA. As a result, 4- $Y$ was significantly higher than all the rest (the main effect of hue in word condition, $F$ [1.39, 54.18] $=19.96, p<.001$; in face condition: $F[1.49,57.97]=22.78, p<.001)$. Surprise: the distribution was bipolar in the word condition. The highest frequency of the word condition was $25.83 \%$ at $4-Y$ and the second highest frequency was $25.50 \%$ at $5-R$. Their values are very close as shown. Repeated-measures ANOVA revealed main effects of brightness, $F(1.91,74.56)=30.88, p<.001$, and of hue, $F(3.23,126.05)=16.73, p$ 
$<.001$. Interaction brightness $\times$ hue was also significant, $F(3.79,147.88)=18.311, p<.001$. In pairwise comparisons for brightness, brightnesses 4 and 5 were significantly higher than the remaining brightness degrees. Post hoc analyses were performed on brightnesses 4 and 5 by one-way repeated-measures ANOVA. In the results of brightness 4, 4- $Y$ was significantly higher than all the rest (the main effect of hue: $F[1.76,68.56]=28.98, p$ $<.001$ ), and in the results of brightness $5,5-R$ was significantly higher than all the rest but $5-Y$ (the main effect of hue: $F[1.76,68.78]=11.41, p<.001)$. In the face condition, the mode was at the same $4-Y$ as the word condition, and its color response was $36.57 \%$. The distribution dispersed wider than in the word condition. Repeated-measures ANOVA revealed main effects of brightness, $F(3.05,118.99)=19.62, p<.001$, and of hue, $F(4.88$, 190.43,)=10.11, p<.001$. Interaction brightness $\times$ hue was also significant, $F(6.71,261.68)=8.33, p<.001$. In pairwise comparisons for brightness, brightness 4 was significantly higher than the remaining brightness degrees. Post hoc analyses were performed on brightness 4 by one-way repeated-measures ANOVA. As a result, 4- $Y$ was significantly higher than all the rest (the main effect of hue: $F[1.97,76.92]=15.90, p<.001)$. No emotion: The coefficient correlation value was lower $(r(128)=.450, p<.001)$ than anger, joy and surprise. As shown the coefficient correlation value, although the shapes of graphs are different, there are some common features between conditions. Concretely, selected color ranges were common, and the modes were at white, $1-B / W$ in both conditions (word: 38.25\%; face: 14.25\%). These graphs in emotion (a)-(d) have a clear peak in each emotion. Moreover, in the face condition, a series of low rises appears in bright area from hue $Y$ to $r P$. This area seems skin-like colors distribution. These distributions of skin-like colors could make the peak lower. Repeated-measures ANOVA revealed main effects of brightness (word: $F[3.58,139.51]=17.60, p<.001$; face: $F$ [3.09, 12.42] $=20.32, p<.001$ ) and of hue in both conditions (word: $F[2.61,101.89]=120.38, p<.001$; face: $F[3.98,155.12]$ $=8.63, p<.001$ ). Interaction brightness $\times$ hue was also significant in both conditions (word: $F[4.45,173.41]=12.80$, $p<.001$; face: $F[9.59,373.87]=3.27, p=.001$ ). In pairwise comparisons of both conditions for brightness, commonly brightness 1 was significantly higher than the remaining brightness degree. Post hoc analyses were performed on brightness 1 by one-way repeated-measures ANOVA. As a result, $1-B / W$ was significantly higher than all the rest in the word condition (the main effect of hue, $F[1.21,47.18]=35.90, p<.001$ ), while $1-B / W$ was not significantly higher in the face condition (the main effect of hue, $F[3.69,143.84]=5.12, p=.001$ ).

Emotion (e)-(g) in Fig. 4, coefficient correlation values for color response are relatively lower than emotion (a)-(d). We depict the detail of results for each emotion as below. Sadness: Based on the results, the word conditions are weakly related to the face conditions, $r(128)=.316, p<.001$. There are not any clear peaks, and distributions of both conditions are dispersed. In the word condition, its distribution disperses widely from bright to dark in bluish hues, while in the face condition, its distribution is dispersed not only on brightness but also on hue. Although we found that their distributions are in bluish hues in common, a series of low rises appears in bright area from $Y$ to $r P$, skin-like colors distribution in the face condition. Accordingly, the coefficient correlation value on hue becomes high $\left(r_{h}(11)=.620, p<.05\right)$, and it demonstrated high similarity for hue distribution between conditions. Repeated-measures ANOVA revealed main effects of brightness in the face condition, $F$ $(5.45,212.57)=2.92, p=.012$, and of hue in both conditions (word condition: $F[3.21,125.31]=16.73, p<.001$; face condition: $F[4.37,170.23]=11.05, p<.001$ ). In pairwise comparisons of both conditions for hue, commonly $B$ was significantly higher than the remaining hues. Disgust and fear: the graphs are totally different between conditions in both emotions, thus, the word conditions are very weakly related to the face conditions (disgust: $r(128)=-.186, p<.05$; fear: $r(128)=-.030, p=.734$ ). Why is the correlation coefficient low between conditions in disgust and fear? In advance, using questionnaires, we examined which emotions were read in the schematic faces. From the results (cf. Table 3 of the Appendix.), faces with high correlation were read properly as we designed (anger as anger: 86.96\%; joy as joy: 90.91\%; surprise as surprise: 86.96\%; sadness as sadness: 62.07\%). However, the faces with low correlation are not read properly as we designed (disgust as disgust: 
36.36\%, fear as fear: $21.43 \%$ ). These face stimuli were read as more variable emotions. Such ambiguous judgments could cause variable color choices by linking to plural emotions in the face conditions. In the face condition, one emotional face linked to plural emotions, whereas, in the word conditions, one emotional word linked to one emotion. Consequently, word condition's distribution could become different from face condition's distribution in disgust and in fear. For disgust, repeated-measures ANOVA revealed main effects of brightness in both conditions (word: $F[2.29,89.45]=24.03, p<.001$; face: $F[5.48,213.63]=3.41, p=.004$ ), and of hue in both conditions (word: $F[5.54,216.19]=5.51, p<.001$; face: $F[6.31,246.19]=3.83, p<.001$ ). Interaction brightness $\times$ hue was also significant in both conditions (word: $F[11.79,459.725]=2.34, p=.007$; face: $F[13.97$, $544.62]=2.03, p=.015$ ). In pairwise comparisons for brightness, brightnesses 8,9 and 10 were significantly higher than most of the remaining brightness degrees in the word condition, while in the face condition, brightness 5 was significantly higher than brightnesses 9 and 10, and brightnesses 4 and 6 were significantly higher than brightness 10. For fear, repeated-measures ANOVA revealed main effects of brightness in both conditions (word: $F[4.74,184.96]=11.38, p<.001$; face: $F[6.28,244.91]=3.455, p=.002$ ), and of hue in both conditions (word: $F[5.31,206.98]=6.25, p<.001$; face: $F[6.513,254.02]=4.86, p<.001$ ). Interaction brightness $\times$ hue was also significant in the word conditions, $F(13.73$. 535.58) $=1.94, p=.021$. In pairwise comparisons for brightness, brightnesses 9 and 10 were significantly higher than most of the remaining brightness degrees in the word condition, while in the face condition, brightness 5 was significantly higher than brightness 2 . Thus, for disgust and fear, the differences of brightness between conditions were found.

We analyzed the association between hue and emotion, by using the results of color response indicated by CIE value (x, y). We call the value (x,y) hue value in our analysis. The general average of CIE values was calculated from the weighted average of weighted color responses of individual participants. Fig. 5 shows general mean of weighted frequency of hue values for each emotion in each condition. In these charts, circle markers indicate focal colors' hue values ( $W$ as white: [.352, .332]; $P$ as purple: [.296, .183]; $B$ as blue: [.177, .191], $G$ as green: [.256, .449], $Y$ as yellow: [.458, .466]; $O$ as orange: [.574, .385]; $R$ as red: [.614, .321]) and diamond marks indicate mean hue values of color response for each emotion. Each emotion is abbreviated as follows, noe is no emotion; ang is angry; dis is disgust; fea is fear; sad is sadness; sur is surprise. We use the charts to understand relative shifts from achromatic hue, white as standard point. In Fig. 5, the chart (a) is word condition and chart (b) is face condition. Between conditions, these charts show similar tendencies though a degree of each shift from white is different. For example, it is found that the anger's hue values shift to $R$ on both conditions, and the surprise's hue values shift to $Y$ in both conditions. For sadness, the hue values shift to $B$ in both conditions. According to its high correlation on hue, it is natural that there is a common tendency, while skin-like colors were selected additionally in the face condition. For fear and disgust, distribution spreads over varied hues, therefore, it is difficult to find a shift tendency on hue. However, fear and disgust slightly shift to $P$, whereas, for disgust alone, face condition's value is closer to $1-O$ as a facial color than in word condition's. This could be caused by pale orange colors selected as facial colors again. Thus, hue values of each emotion indicate some tendencies between colors and emotions. In addition to it, in the face condition, the general distribution is closer to $W$ or rather $1-O$ as a facial color than in the word condition. 
(a) Anger $(r=.966, p<.001)$

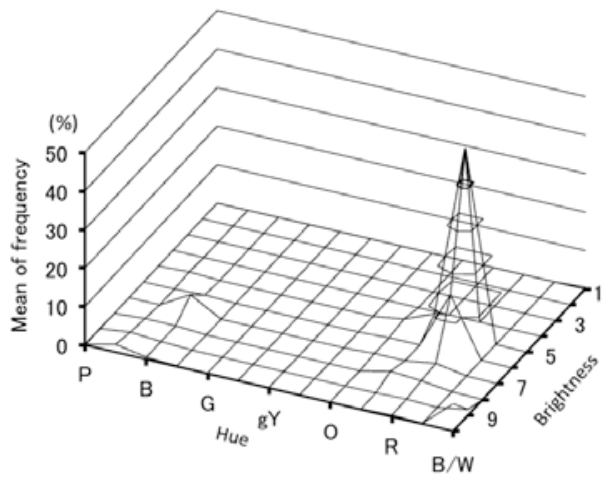

(b) $\operatorname{Joy}(r=.882, p<.001)$

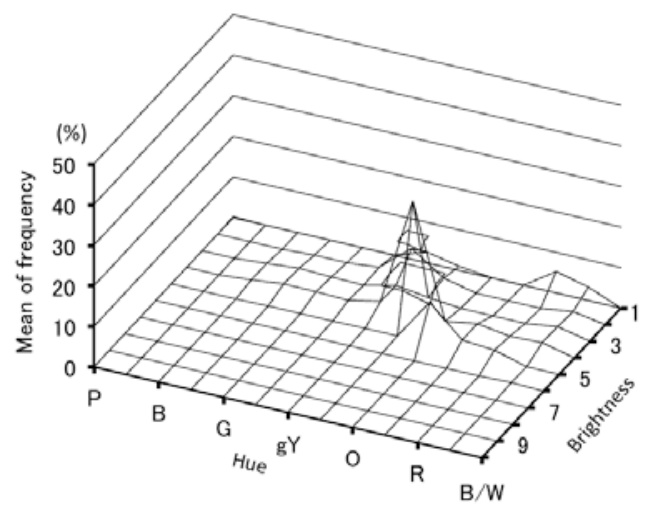

(c) Surprise $(r=.695, p<.001)$

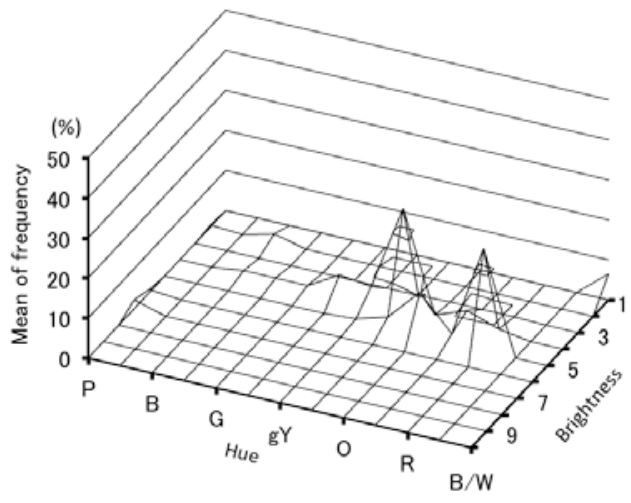

(d) No emotion $(r=.450, p<.001)$

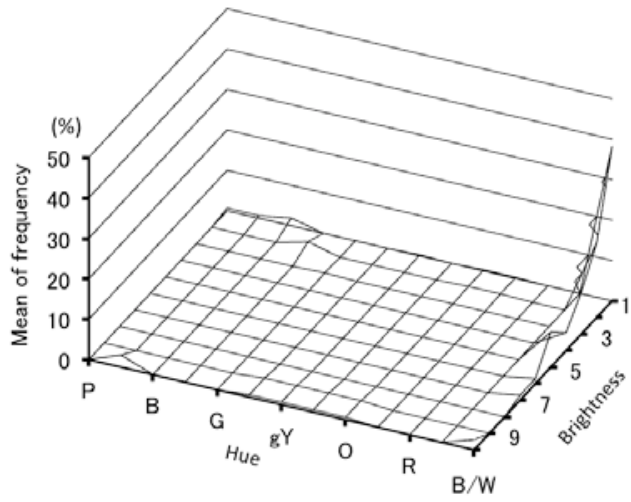

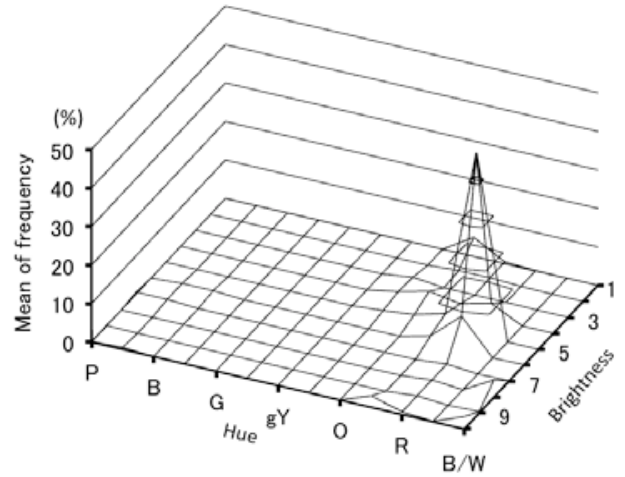
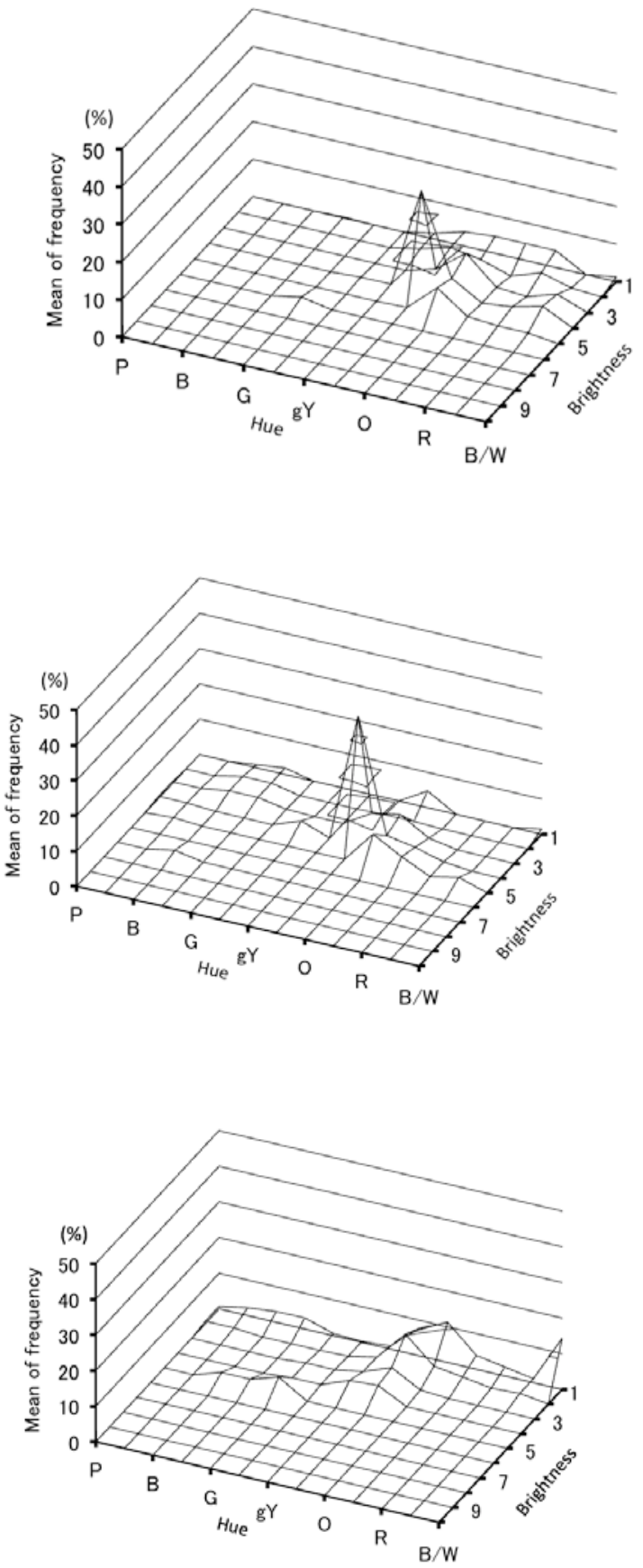
(e) Sadness $\left(r=.316, p<.001\right.$; correlation on hue $\left.r_{h}=.620, p=.024\right)$
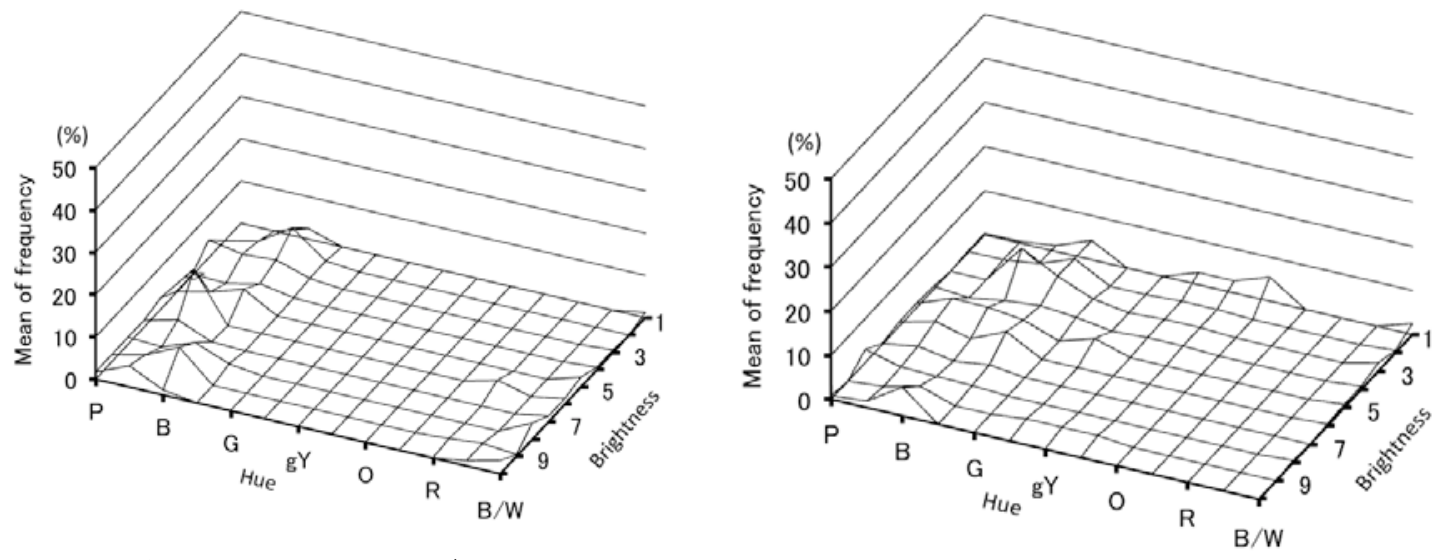

(f) Disgust $\quad(r=-.186, p=.034)$
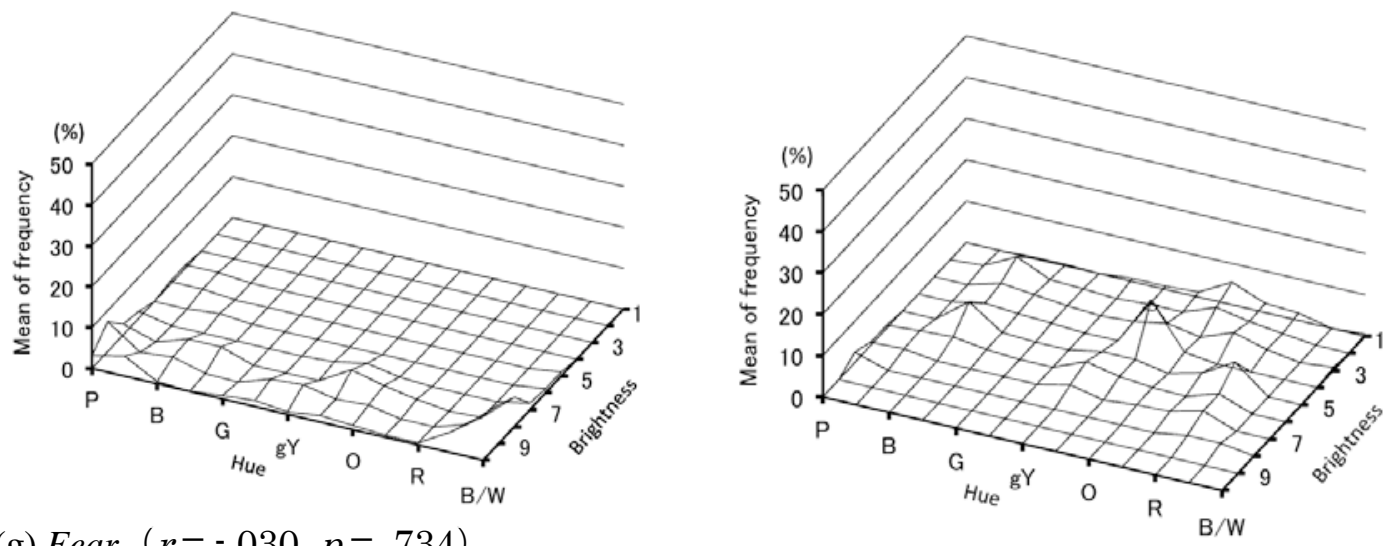

(g) Fear $(r=-.030, p=.734)$
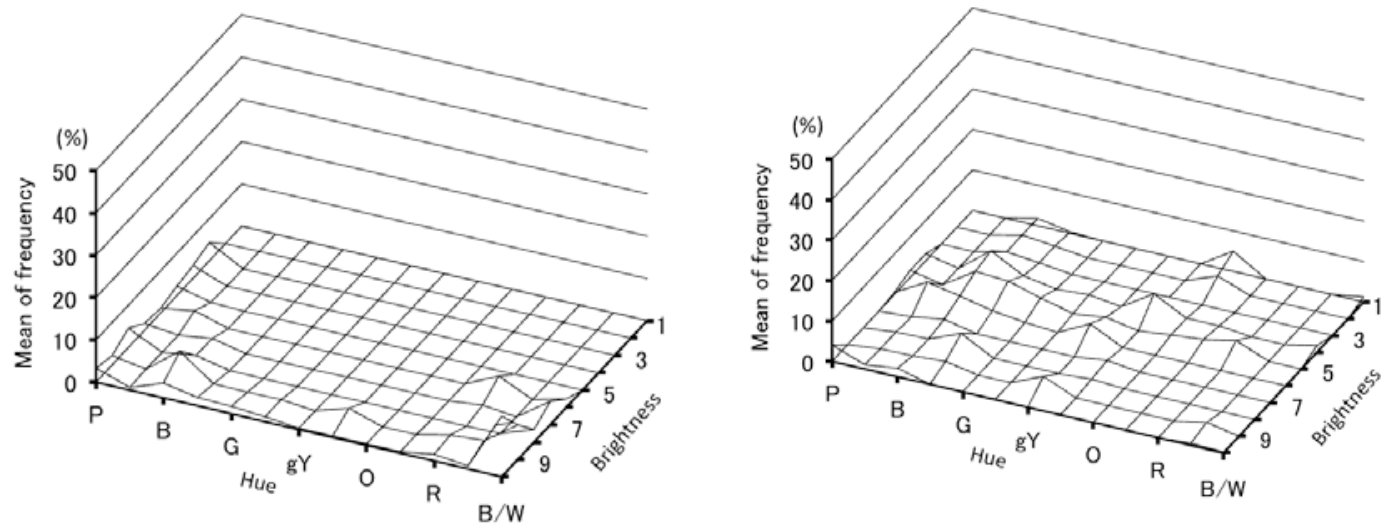

Fig. 4. The results of color response for each emotion in two conditions.

The left columns show word conditions and the right columns show face conditions. Each line gives the results of emotion, (a) anger, (b) joy, (c) surprise, (d) no emotion, (e) sadness, (f) disgust and (g) fear. The vertical axis (z-axis) indicates the average of weighted frequency on color response, and two horizontal axes (the x-axis: left to right; the y-axis: front to back) indicate the array of color samples. The $\mathrm{x}$-axis indicates brightness. 
(a) Word condition (first participant group)

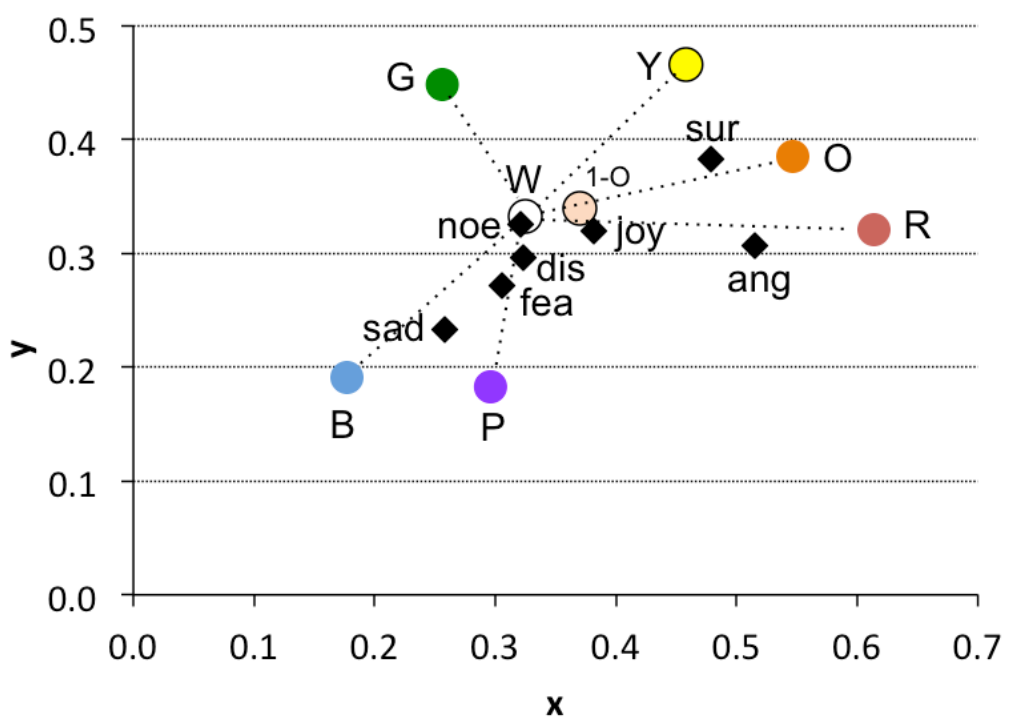

(b) Face condition (second participant group)

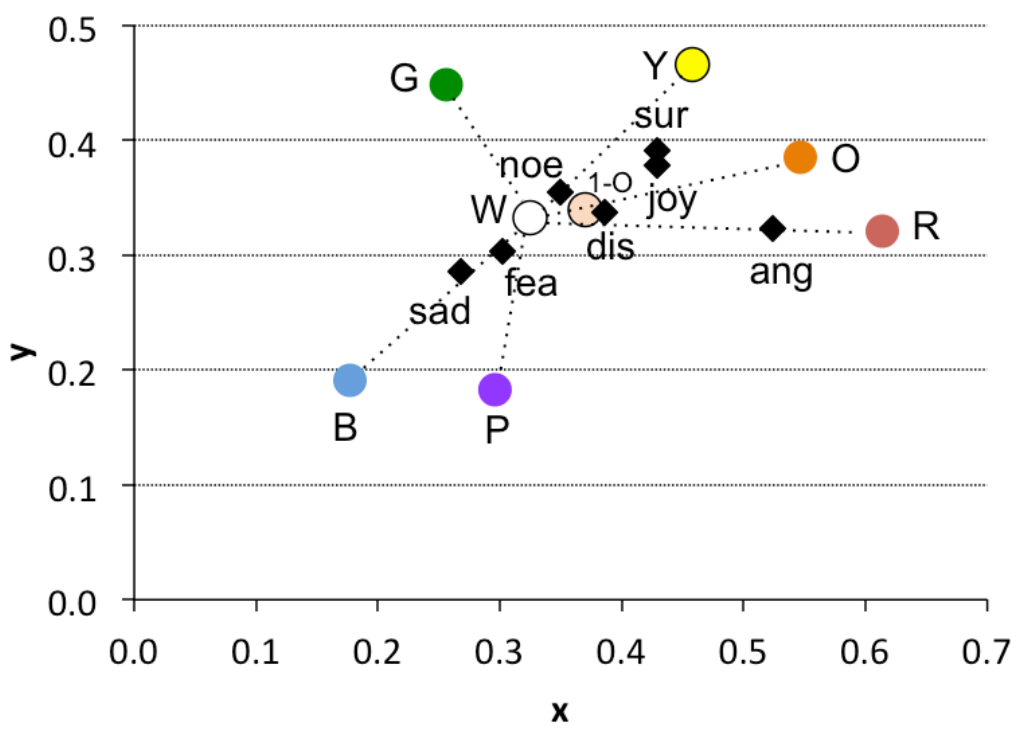

Fig. 5. Mean of hue values in CIE value (x, y) for each emotion.

The general means of selected hue values are shown for each emotion in each condition. In these charts, circle markers indicate focal colors' hue values ( $W$ as white; $P$ as purple; $B$ as blue; $G$ as green; $Y$ as yellow; $O$ as orange; $R$ as red; $1-O$ is a color sample on the color palette as a facial color; and diamond marks indicate mean hue values of color response for each emotion). Each emotion is abbreviated as follows, noe is no emotion; ang is angry; dis is disgust; fea is fear; sad is sadness; sur is surprise. (a) is word condition and (b) is face condition.

\section{Association between Brightness and Emotions}

Although the difference in related brightness due to emotion or to condition are shown above, two graphs are given to illustrate the differences by comparing the average values of brightness of each emotion. The general average of CIE values was calculated from the weighted average of weighted color responses of individual participants. Fig. 6 depicts the mean of CIE value $(\mathrm{Y})$ for each emotion on each condition to find out the association between emotion and brightness. Brightness values of sadness, anger, fear and disgust, as negative emotions, were lower than the value of joy, surprise and no emotion. Thus, it is concluded that negative emotions are associated with darker 
colors, and that positive emotions are associated with brighter colors. These associations support the results of the research on children's drawing. ${ }^{32}$ The associations appeared clearer in the word condition, since the values of negative emotions in word condition are located downward relative to the face condition, and the range of all emotions' values are wider than face condition. We investigated the reason why the differences between conditions occurred. According to the answer sheets of word condition, emotional words evoked many kinds of objects as a trigger to imagine colors. However, schematic faces limit participants to evoke just face and possibly a few objects. This difference of diversity for evoking objects might influence the range of brightness.

(a) word condition (first participant group)

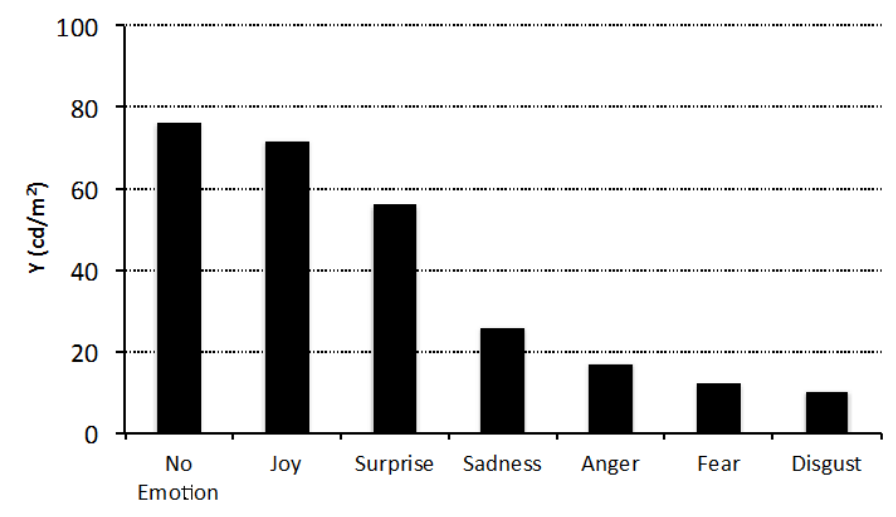

(b) face condition (second participant group)

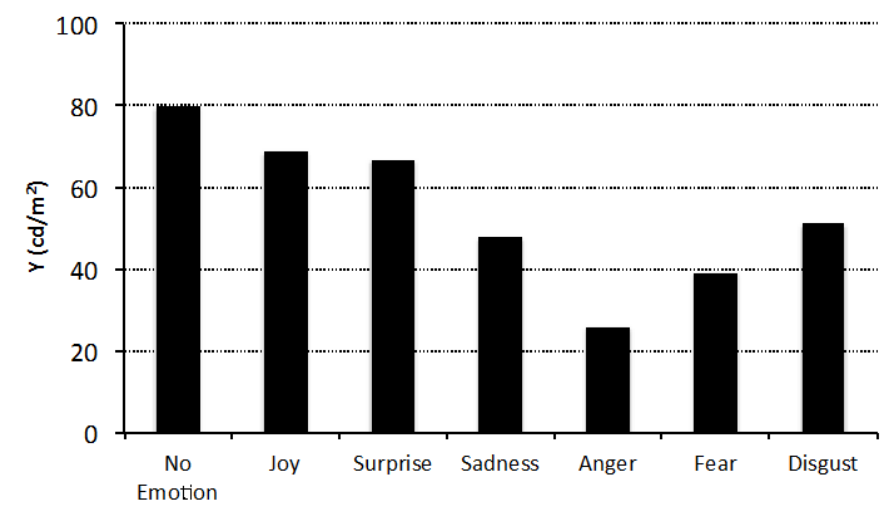

Fig. 6. Mean of selected colors in CIE value $(\mathrm{Y})$ as brightness for each emotion.

(a) is word condition (first participant group), and (b) is face condition (second participant group). 


\section{Discussion}

The association between color and emotion were investigated using seven basic emotional words and a set of schematic faces corresponding to the emotions. In the results, certain associations between colors and emotions were found in both conditions, and the tendencies of associations were different among emotions. Anger, joy, surprise, sadness and no emotion were connected to particular colors. However, the distributions of sadness were spread among the bluish colors. The color response depicted a correspondence to bluish hues rather than to specific color samples. This tendency was similar in both conditions. However, in the schematic face condition, the color responses of all emotions were increased in the skin-colored samples*². Thereby, the context of face could elicit the color responses.

To begin with, we discuss the response tendencies for the emotions for which were found associations with specific colors. Anger was associated with a specific color sample, namely, 5- $R$, which is the most saturated red, in both conditions. Anger being associated with red supports the results of previous studies. ${ }^{10,20}$ Why did this tendency appear in common between two conditions? The responses to the questionnaire are found in Table 3 of the Appendix, in which it can be seen that for the word condition, comments associating it with a face red with anger were listed most as triggers for the chosen color. That is to say, the angry face was a common image between the two conditions.

Further, emotional words and schematic faces are more abstracted than actual faces. It is known that memory color is judged differently from actual color in terms of physical features. Elicited targets of a higher abstractness could recall a focal color that is easily perceived biologically. ${ }^{33}$ Therefore, when a face red with anger is retrieved from memory, the redness could shift to a more saturated color. Accordingly, common tendencies between the two conditions centered on the most saturated red, 5- $R$, caused the similarity in responses. Joy was associated with multiple colors, however, the mode was fully saturated yellow, 4- $Y$, in both conditions. The diversity of color response supports Byrne's result (1983) ${ }^{10}$ that a variety of colors were selected for happiness. Why were color responses centered at yellow? Light was recalled most as a trigger to imagine the color for joy (cf. Table 3 in the Appendix). The light was associated with yellow, and it led to the responses of the saturated yellow, 4-Y. In surprise, the mode of color response was fully saturated yellow, 4-Y. Table 3 in the Appendix, which shows responses to a questionnaire for the word condition, jagged shapes from cartoons and illustrations, traffic signs, and light were listed as triggers for the color for surprise. Since the typical or symbolic color of these is yellow, yellow was the most common response. Besides, white was also often given as a response. In the Japanese idiom, ganmen sohaku (one's face turns to bluish white) and atama ga massiro ni naru (to become white in one's head) are used to express surprise. These expressions including white may have prompted the response of white. Sadness was associated with blue and bluish colors. In Table 3 of the Appendix, tears, rain, and the sea bed are listed as triggers for sadness. These all relate to water. Furthermore, buru ni naru (to feel blue) in a Japanese idiom expresses a sad mood. Hence we considered that these idioms could affect the responses. For no emotion, the mode of the color response was white $(1-B / W)$. The other emotions were mostly associated with chromatic colors, whereas no emotion was associated with an achromatic color. Emotion causes a change of oxygen in the blood, however, the status of no emotion brings no change in the blood. No emotion means no mood or no affect. Hence the concept of nothing may be connected to white, as it is achromatic.

When people become angry, their faces turn red due to increased blood flow to the skin. There is a rational association between anger and red. This association is consistent with Mollon's hypothesis (1989) ${ }^{7}$ that color vision in primates was selected to discriminate the spectral modulations on the skin of conspecifics, presumably for the purpose of discriminating emotional states, socio-sexual signals and threat displays. ${ }^{7,8}$ Further, for joy,

\footnotetext{
*2 In this study, the participants were Japanese. Therefore, skin-colored samples mean the skin color of xanthous.
} 
pinkish colors and an orange of focal color were given frequently as responses. We considered that our experience seeing people's blushing faces in their joy would cause these responses. The association between joy and redness supports the results of Suzuki and Koyatsu (1996). ${ }^{9}$

However, ganmen sohaku originates from an actual facial expression of surprise and other negative statuses. The face turns pale due to impaired redness caused by decreasing oxygen in the blood. Hence we consider that the association between the actual facial expression and facial color may reflect the response of associated color even for the abstract stimuli used in this study. Moreover, we must consider the connection to sensations. There were many responses associated with heat and high energy status in the word condition (cf. Table 3 of the Appendix; e.g., fire, flame, heat, boiling, a pot red with heat), and these answers are consistent with the hue-heat hypothesis that dominant light frequencies toward the red end are connected to warmness. ${ }^{5}$ Furthermore, positive emotions associated with relatively bright colors and negative emotions are associated with relatively darker colors as a result. For example, fear and disgust were not associated with specific color or hue but with low brightness colors. This result supports Schirillo (2001), ${ }^{34}$ who investigated colors in children's drawings, and Saito (1996), ${ }^{17}$ who performed a cross-cultural study of color preference.

Although anger and joy are associated with redness, a fully saturated red color sample was given most for anger, while pinkish colors and orange were given as responses for joy. In fact, the faces in both these emotions show blushes. Why are there differences in brightness and saturation between anger and joy? According to the affect grid with the bipolar dimension scales arousal-sleepiness and pleasure-displeasure, ${ }^{35}$ while both anger and happiness are located in arousal, anger is located in displeasure and happiness is located in pleasure. We suppose that pleasure/displeasure may cause differences in brightness and saturation, and the difference of brightness between anger and joy should be consistent with the result that positive/negative emotions are associated with brightness. ${ }^{16,17,18}$

The association between emotions and brightness are presumed not only in a facial expression but also by innate human nature. Since the cone of a photoreceptor perceives color in photopic vision, color relates to closely light. It is said that more people become depressed during the season of polar night in the polar regions. Arendt (2012) ${ }^{36}$ suggested that time exposed to light relates to the circadian rhythms and to physical or mental health. Accordingly, we propose an association between mood or rather emotion and brightness as well as color.

Fig. 6 shows that the color response in the schematic face condition tended to have a distribution in higher brightness. We consider three reasons for this tendency. The first is the latitude of color in the human skin. Since our xanthous skin color is rather bright, low brightness can be eliminated for facial color. The second is discrimination of the contour of the schematic faces. Since facial expressions were described by black lines, the greater brightness may be needed to discriminate the black lines. The third is the effect of popularized expressions in emoji of mobile terminals, games, cartoons and illustrations. These may have influenced the color responses.

\section{Conclusion}

In this study, we investigated how colors are evoked by emotions by using seven basic emotional words and a set of schematic faces corresponding to these words. Associations between color and emotion were found in both stimuli conditions in anger, joy, surprise, sadness and no emotion. Moreover, positive emotions tended to be associated with greater brightness and more saturated colors and negative emotions tended to associate with lower brightness colors.

We presumed the color response would be different depending on different abstraction levels. As a result, color responses between the word condition and face condition were strongly related in anger, joy, surprise, and moderately related in no emotion. Moreover, for sadness, we found the association with blue hue not in a specif- 
ic color sample in either condition. That is, we could not find the differences between abstraction level of stimuli. However, the context effect of the face was found. Taking as our perspective, a view on the origin of human color vision that our trichroma originated from discriminating spectral modulations on the skin, ${ }^{7,8}$ we hold that spectral modulations on the skin that are part of facial expression is used as an important social signal. Thereby the association between color and emotion appeared in both conditions with a different level of abstraction for the stimuli. The results of the present study are consistent at some points with previous studies. However, using an original color palette with much more samples than in previous studies, we found significant, subtle differences in the colors associated with the emotions and between conditions.

\section{References}

1. Manav, B., (2007). Color-Emotion Associations and Color Preferences: A case Study for Residences. Color Research and Applications, 32, 144-151.

2. Kawamoto, N., \& Soen, T. (1993). Objective evaluation of color design: II. Color Research and Application, 18, 4, 260-266.

3. Ou, L. C., Luo, M. R., Woodcock, A., Wright, A., (2004). A study of colour emotion and colour preference. Part I. Colour emotions for single colours. Color Researth and Application, 29, 232-240.

4. Gao, X.-P., Xin, J. H., Sato, T., Hansuebsai, A., Scalzo, M., Kajiwara, K., Billger, M. (2007). Analysis of cross-cultural color emotion. Color Research \& Application, 32, 3, 223-229.

5. Bennet, C. A., \& Rey, P. (1972). What's So Hot About Red? Human Factors, 14, 2, 149-154.

6. Mahnke, F., (1996). Color, Environment, Human Response, New York: Van Nostrand Reinhold; 1996.

7. Mollon, J. D. (1989). "tho' she kneel'd in that place where they grew..." The uses and origins of primate colour vision. Journal of Experimental Biology, 146, 21-38.

8. Changizi, M.A., Zhang, Q., and Shimojo, S., (2006). Bareskin, blood and the evolution of primate color vision, Biology Letters, 2, 217- 221.

9. Suzuki, T., \& Koyatsu, T., (1998). The Study of the Effect of the Face Color upon the Recognition of the Emotion Caused by the Facial Expression, The Journal of the Color Science Association of Japan, 22, 2, 45-52.

10. Byrnes, D. A., 1983. Color Associations of Children. The journal of Psychology, 113, 247-250.

11. Terwogt, M. M., \& Hoeksma, J.B., (2001). Colors and Emotions: Preferences and Combinations. The Journal of General Psychology, 122, 1, 5-17.

12. Adams, F. M., \& Osgood, C. E. (1973). A Cross-Cultural Study of the Affective Meanings of Color. Journal of Cross-Cultural Psychology, 4, 2, 135-156.

13. Schaie, K. W., (1962a). SCALING THE ASSOCIATION BETWEEN COLORS AND MOODTONES. The American Journal of Psychology, 74, 266-273.

14. Schaie, K. W., (1962b). A Q-sort study of color-mood association, Journal of Projective Techniques, 25, 341-346.

15. Wexner, L., (1954). The Degree to Which Colors (hues) are associated with moodtones. Journal of Applied Psychology, 38, 432-455.

16. Cimbalo, R. S., Beck, K. L., \& Sendziak, D. S. (1978). Emotionally Toned Pictures and Color Selection for Children and College Students. The Journal of Genetic Psychology, 133, 2, 303- 
304.

17. Saito, M., (1996). Comparative studies on color preferences in Japan and other Asian regions with special emphasis on the preference for white. Color Research and Application, 21, 1, 35-49.

18. Hemphill, M. (1996). A note on adults' color-emotion associations. The Journal of Generic Psychology, 157, 3, 275-280.

19. Kaya, N., \& Epps, H. H. (2004). Relationship between color and emotion: A study of college students. College Student Journal, 38, 3, 396-405.

20. Mashiko, Y. \& Saito, M., (2008). Effect of Facial Expression to Image Color of Person. The Journal of the Color Science Association of Japan, Supplement, 32, 60-61.

21. Ekman, P., (1973). Emotion in the human face. Cambridge, MA: Cambridge University Press.

22. Paul Ekman, W. V. F. (1969). The Repertoire of Nonverbal Behavior Categories Origins, Usage, and Coding. Semiotica, 1, 1, 49-98.

23. Eastwood, J. D., Smilek, D., \& Merikle, P. M. (2001). Differential attentional guidance by unattended faces expressing positive and negative emotion. Perception \& Psychophysics, 63, 6, $1004-13$.

24. Fox, E., Russo, R., \& Dutton, K. (2002). Attentional bias for threat: Evidence for delayed disengagement from emotional faces. Cognition and Emotion, 16(3), 355-379.

25. Nothdurft, H. (1993). Faces and facial expressions do not pop out. Perception, 22, 1287-1298.

26. Öhman, A., Lundqvist, D., \& Esteves, F. (2001). The Face in the Crowd Revisited: A Threat Advantage With Schematic Stimuli. Journal of Personality and Social Psychology, 80, 3, 381396.

27. Purcell, Dean G., Alan L. Stewart, and Richard B. Skov. (1996). It takes a confounded face to pop out of a crowd. Perception 25, 9, 1091-1108.

28. Tipples, J., Atkinson, A. P., \& Young, A. W. (2002). The eyebrow frown: A salient social signal. Emotion, 2(3), 288-29.

29. White, Murray. (1995). Preattentive analysis of facial expressions of emotion. Cognition \& Emotion, 9, 5, 439-460.

30. Hansen, C. H., \& Hansen, R. D. (1988). Finding the face in the crowd: an anger superiority effect. Journal of Personality and Social Psychology, 54, 6, 917-24.

31. Lundqvist, Daniel;Esteves, Francisco;Ohman, A. (2004). The face of wrath: The role of features and configurations in conveying social threat. Cognition and Emotion, 18, 2, 161-182.

32. Boytis, C. J., \& Varghese, R. (n.d.). Children's Emotional Associations With Colors. The Journal of Genetic Psychology, 155, 1, 77-85.

33. Ratner, C., \& McCarthy, J. (1990). Ecologically relevant stimuli and color memory. The Journal of General Psychology, 117, 369-377.

34. Schirillo, J.A., (2001). Tutorial on the Importance of Color in Language and Culture. Color Research and Application, 26, 3, 179-192.

35. Russell, J.A., \& Fernandez-Dos, J. M., (1977). The Psychology of Facial Expression. Cambridge University Press.

36. Arendt, J., (2012). Biological rhythms during residence in polar regions. Chronobiology international, 29, 4, 379-394.

\section{Appendix}


Table 1. The CIE values of color samples.

The CIE values of color samples were calibrated by a chromatic photometer, CA-100. The top line indicates hue $(P-B / W)$ and left column (1-10) indicates the brightness and saturation level. In each cell, the value $\mathrm{x}, \mathrm{y}$ at the upper line and $\mathrm{Y}\left(\mathrm{cd} / \mathrm{m}^{2}\right)$ at down line. On the color pallette, the columns were indicated 1-13, and the lines were indicated A-J.

\begin{tabular}{|c|c|c|c|c|c|c|c|c|c|c|c|c|c|}
\hline & $\mathrm{P}$ & $p B$ & B & $B G$ & $\mathrm{G}$ & yG & $g Y$ & $Y$ & $\mathrm{O}$ & yR & $\mathrm{R}$ & rP & $\mathrm{B} / \mathrm{W}$ \\
\hline \multirow{2}{*}{1} & $(.32, .30)$ & $(.30, .29)$ & $(.29, .32)$ & $(.29, .33)$ & $(.30, .35)$ & $(.34, .38)$ & $(.36, .37)$ & $(.38, .36)$ & $(.37, .34)$ & $(.37, .33)$ & $(.36, .30)$ & $(.35, .29)$ & $(.33, .33)$ \\
\hline & 74.2 & 67.1 & 71.5 & 87.5 & 82.6 & 81.4 & 94.0 & 89.7 & 72.9 & 74.9 & 67.8 & 72.3 & 108.0 \\
\hline \multirow{2}{*}{2} & $(.32, .27)$ & $(.29, .25)$ & $(.27, .31)$ & $(.27, .33)$ & $(.29, .36)$ & $(.35, .40)$ & $(.38, .39)$ & $(.38, .38)$ & $(.40, .35)$ & $(.36, .34)$ & $(.39, .30)$ & $(.35, .29)$ & $(.32, .33)$ \\
\hline & 62.2 & 53.1 & 68.0 & 80.2 & 82.5 & 85.5 & 92.4 & 91.4 & 71.8 & 64.9 & 57.8 & 65.8 & 92.0 \\
\hline \multirow{2}{*}{3} & $(.31, .25)$ & $(.26, .23)$ & $(.24, .27)$ & $(.25, .33)$ & $(.28, .39)$ & $(.35, .43)$ & $(.38, .42)$ & $(.41, .40)$ & $(.43, .36)$ & $(.44, .34)$ & $(.42, .30)$ & $(.37, .26)$ & $(.32, .32)$ \\
\hline & 46.6 & 42.0 & 52.2 & 68.0 & 77.0 & 77.9 & 82.4 & 82.7 & 63.1 & 49.0 & 49.4 & 52.9 & 61.8 \\
\hline \multirow{2}{*}{4} & $(.30, .22)$ & $(.24, .19)$ & $(.21, .24)$ & $(.23, .32)$ & $(.27, .41)$ & $(.35, .48)$ & $(.40, .45)$ & $(.46, .47)$ & $(.48, .38)$ & $(.50, .35)$ & $(.48, .30)$ & $(.39, .25)$ & $(.32, .32)$ \\
\hline & 39.1 & 30.3 & 41.9 & 66.6 & 70.5 & 73.6 & 83.1 & 85.3 & 54.1 & 41.3 & 39.2 & 44.7 & 47.5 \\
\hline \multirow{2}{*}{5} & $(.27, .19)$ & $(.20, .15)$ & $(.18, .21)$ & $(.24, .32)$ & $(.27, .39)$ & $(.36, .44)$ & $(.39, .43)$ & $(.47, .45)$ & $(.55, .39)$ & $(.57, .38)$ & $(.61, .32)$ & $(.40, .23)$ & $(.32, .31)$ \\
\hline & 24.2 & 17.6 & 27.5 & 45.8 & 48.8 & 48.7 & 56.1 & 62.3 & 40.3 & 29.9 & 19.6 & 31.4 & 37.1 \\
\hline \multirow{2}{*}{6} & $(.28, .19)$ & $(.20, .15)$ & $(.18, .21)$ & $(.21, .30)$ & $(.26, .45)$ & $(.35, .51)$ & $(41, .48)$ & $(.46, .45)$ & $(.54, .40)$ & $(.46, .34)$ & $(.55, .30)$ & $(.39, .23)$ & $(.31, .31)$ \\
\hline & 17.40 & 11.60 & 18.10 & 34.20 & 37.00 & 36.10 & 44.40 & 40.40 & 23.20 & 23.10 & 16.20 & 19.70 & 26.60 \\
\hline \multirow{2}{*}{7} & $(.30, .18)$ & $(.22, .16)$ & $(.18, .19)$ & $(.21, .30)$ & $(.25, .39)$ & $(.36, .48)$ & $(.40, .46)$ & $(.44, .44)$ & $(.49, .39)$ & $(.51, .36)$ & $(.48, .28)$ & $(.37, .22)$ & $(.31, .31)$ \\
\hline & 12.7 & 11.2 & 13.4 & 26.2 & 27.7 & 30.0 & 32.2 & 31.5 & 20.3 & 16.9 & 13.9 & 16.0 & 19.1 \\
\hline \multirow{2}{*}{8} & $(.29, .19)$ & $(.22, .16)$ & $(.19, .22)$ & $(.21, .30)$ & $(.25, .40)$ & $(.36, .49)$ & $(.41, .46)$ & $(.45, .44)$ & $(.49, .40)$ & $(.53, .36)$ & $(.49, .29)$ & $(.37, .22)$ & $(.31, .31)$ \\
\hline & 10.4 & 71.5 & 10.9 & 19.1 & 17.9 & 18.2 & 23.4 & 20.3 & 13.2 & 10.3 & 8.69 & 10.4 & 11.9 \\
\hline \multirow{2}{*}{9} & $(.28, .21)$ & $(.24, .19)$ & $(.21, .24)$ & $(.22, .32)$ & $(.26, .37)$ & $(.36, .43)$ & $(.38, .44)$ & $(.40, .42)$ & $(.44, .38)$ & $(.46, .34)$ & $(.43, .29)$ & $(.34, .23)$ & $(.32, .31)$ \\
\hline & 7.78 & 6.32 & 8.63 & 12.9 & 12.0 & 12.3 & 14.9 & 14.2 & 9.83 & 8.02 & 7.02 & 7.83 & 5.26 \\
\hline \multirow[t]{2}{*}{10} & $(.28, .21)$ & $(.23, .18)$ & $(.21, .23)$ & $(.23, .32)$ & $(.26, .39)$ & $(.34, .45)$ & $(.39, .44)$ & $(.41, .42)$ & $(.45, .38)$ & $(.48, .34)$ & $(.44, .30)$ & $(.35, .24)$ & $(.31, .29)$ \\
\hline & 4.43 & 3.35 & 4.06 & 8.07 & 6.60 & 6.35 & 8.82 & 7.46 & 4.68 & 4.38 & 3.62 & 4.02 & 0.38 \\
\hline
\end{tabular}


Table 2. Results of emotion judgment for schematic faces.

At the end of the experiment, 20 participants responded questionnaires about what emotions he/she felt for each schematic face by multiple selections of the seven basic emotions. The Results are shown by frequencies and parentheses indicate a percentage of frequency.

\begin{tabular}{crrrrrrr}
\hline & NOE & \multicolumn{1}{c}{ JOY } & \multicolumn{1}{c}{ SAD } & \multicolumn{1}{c}{ ANG } & \multicolumn{1}{c}{ DIS } & \multicolumn{1}{c}{ SUR } & \multicolumn{1}{c}{ FEA } \\
\hline no emotion & $\underline{20(69.0)}$ & $0(0.0)$ & $4(13.8)$ & $0(0.0)$ & $0(0.0)$ & $1(4.4)$ & $0(0.0)$ \\
joy & $0(0.0)$ & $\underline{20(90.9)}$ & $0(0.0)$ & $0(0.0)$ & $0(0.0)$ & $2(8.7)$ & $0(0.0)$ \\
sadness & $1(3.5)$ & $0(0.0)$ & $\underline{18(62.1)}$ & $0(0.0)$ & $5(15.2)$ & $0(0.0)$ & $8(19.1)$ \\
anger & $0(0.0)$ & $0(0.0)$ & $0(0.0)$ & $\underline{20(87.0)}$ & $1(3.0)$ & $0(0.0)$ & $0(0.00$ \\
disgust & $1(3.5)$ & $0(0.0)$ & $1(3.5)$ & $3(13.0)$ & $\underline{12(36.4)}$ & $0(0.0)$ & $9(21.4)$ \\
surprise & $3(10.34)$ & $0(0.0)$ & $0(0.0)$ & $0(0.0)$ & $3(9.1)$ & $\underline{20(87.0)}$ & $10(23.8)$ \\
fear & $0(0.0)$ & $0(0.0)$ & $1(3.5)$ & $0(0.0)$ & $5(15.2)$ & $0(0.0)$ & $\underline{9(21.4)}$ \\
other & $4(13.79)$ & $2(9.1)$ & $5(17.2)$ & $0(0.0)$ & $7(21.2)$ & $0(0.0)$ & $6(14.3)$ \\
\hline sum. & $29(100)$ & $22(100)$ & $29(100)$ & $23(100)$ & $33(100)$ & $23(100)$ & $42(100)$ \\
\hline
\end{tabular}


Table 3. Evoked images from each emotional word.

Evoked images from each emotional word to imagine associated colors, which were retrieved from answer sheets and participants' comments. These answers are translated directly to English and Similar English expressions are provided in brackets. The parentheses indicate numbers of answers.

* asterisks are regarded as "a face red with anger".

\begin{tabular}{cl}
\hline $\begin{array}{c}\text { Emotional } \\
\text { word }\end{array}$ & \multicolumn{1}{c}{ Evoked images to image colors } \\
\hline $\begin{array}{c}\text { No emo- } \\
\text { tion }\end{array}$ & $\begin{array}{l}\text { natural taste of the interior and fabrics, Buddhist monk with anatta, } \\
\text { modest state -> no emotion -> no color, nothing is equivalent to white, black hole and white hole, white paper } \\
\text { [blank sheet], to become white in one's head [one's head goes blank], no notion, feeling empty, nearly trans- } \\
\text { parent, a space with no color, my friend's empty gaze, no facial expression (2), a person with no facial ex- } \\
\text { pression, image of myself with no emotion, gears of person looking emotionless, a patient room in medical } \\
\text { ward of psychiatric hospital, a person by my side, }\end{array}$ \\
\hline Anger & $\begin{array}{l}\text { an angry face of a real person, expression of anger (e.g. illustration, comic, pictogram in mobile phone), } \\
\text { *angry face and flame in comic and in illustration, comic expression, *appearance of anger with red face in } \\
\text { comic, background color of text in game for anger situation, idiom: to be red with anger, a rather darker red } \\
\text { ball, *facial color when people get angry (3), angry face of people, *red is angry face of human, a scary face, } \\
\text { flushed face, *a face with having a hemorrhage, appearance of excitement of human, my friend's anger, } \\
\text { blood(2), dreggy and unhealthy blood, color of fire, fire, flame: mainly red and blue, flame, heat, state with } \\
\text { heat, boiling, a pot red with heat, a pot, blackish color for hatred, black: anger which is close to hatred (e.g. } \\
\text { vengeance, obsessiveness), I13 and J13 on the color pallet are exact colors of the emotion, total darkness, } \\
\text { blizzard: mainly blue and a little white }\end{array}$ \\
\hline
\end{tabular}

Sadness expression of sadness (e.g. illustration, comic, pictogram in mobile phone), picture, a pale face of human, a person who look like sad, tears (5), water, rain, drizzle, sea bed is blue, image of coldness, feeling of hilly, idiom: to feel blue, namely called sad emotional state 'blue', fresh and brisk, graduation ceremony, funeral (3), death, darkness, feeling deep, grave, ash, aqua color is for funeral and purple is of Japanese wrapping fabric, the clothes color which my grand mom wore and cried when my grand pa died recently,

Joy light, sun (4), something like sun (e.g. aureola, light), lightness, light color, light image, flower colors, flower (3), flower bunch, song, love, nature, wind, blue sky, freshness, fresh verdure, viridity, spring and summer, image of spring, stuffed toy, pleasure, a face of excited person, a smiley face of person especially female and child, a smiley face (2), wedding ceremony, announcement of successful applicants, colors of favorite food especially fruits, favorite colors as if they remind me happy time, favorite colors of clothes and of small products,

Disgust greenish brown: insects or bugs and humidity and rain cloud, gunky chunk of negativity, snake, crow, waste materials, rubbish, dirtheap with dead bodies of animals and waste, colors of wall in dirty toilet, devil, archenemy, fight, spider, facial color, something cold, a specific dislikable person, a dislikable person, feeling allergic, jealousy, appearance of Chinese characters” 嫌悪 “ evokes impression and colors, connect the word with, darkness, dark, feeling dark, ambiguous dark colors, not clear, dirty colors, look delicious food,

Surprise traffic sign, the attention of traffic sign (2), electric bulb, strong light, light of sun, light and distinct color, eye-catching color, colors used very often in advertisement of sale, curiosity, '!' (2), red '!' in pictogram of mobile phone etc. (3), yellow jaggy shape used in illustration and comic, anthropoid apes such as gorilla and mandrill, to become white in one's head [one's head goes blank, blank out], one's face turns bluish white [to turn pale, white as a sheet], turned pale of facial color, ghost, face expression which a person open his/her eyes widely, "yellow was evoked when I imagined to be surprised", "I imagined a scene which I was surprised", a image of having crashed an object, a cracker to surprise,

Fear expression of fear such as illustration and comic and pictogram, an image of human's face and of whole scene in cold and dark place, background of a picture, colored comic, something unknown, snake, the angel of death, funeral, blood, disease, death, darkness, night (3), gloom, feeling of cold and dark, ocean of trees on Mt. Fuji, nightmare, haunt, color of clothes, a situation which my father gets angry, "I imagined a scene when I was scared". 